\title{
Efeito da adição de escória de alto- forno em compósitos com bambu mossô
}

\section{Effect of adding blast furnace slag to composites with mosso bamboo}

\author{
Luana Cechin ${ }^{1}$, Adalberto Matoski ${ }^{1}$, Adauto José Miranda de Lima ${ }^{2}$, \\ Alessandra Monique Weber ${ }^{1}$, Rosângela Basso Tokarski ${ }^{1}$
}

\footnotetext{
${ }^{1}$ Programa de Pós-Graduação em Engenharia Civil, Universidade Tecnológica Federal do Paraná - UTFPR, R. Dep. Heitor Alencar Furtado, 5000 - Campo Comprido, CEP 81280-340, Curitiba, Paraná, Brasil.

${ }^{2}$ Departamento Acadêmico de Engenharia Civil, Universidade Tecnológica Federal do Paraná - UTFPR, R. Dep. Heitor Alencar Furtado, 5000 - Campo Comprido, CEP 81280-340, Curitiba, Paraná, Brasil.

e-mail: luhcechin@gmail.com, adalberto@utfpr.edu.br, adautojm@gmail.com, alessandramoniqueweber@gmail.com, rosangela.tokarski@ifsc.edu.br
}

\section{RESUMO}

O objetivo desta pesquisa foi avaliar o efeito da adição de diferentes teores escória de alto-forno à carvão vegetal em compósitos com bambu mossô (Phyllostachys pubescens), identificando o grau de compatibilidade entre esses componentes. O grau de compatibilidade foi determinado através do estudo do calor de hidratação nas primeiras 24 h e pela resistência à compressão axial após 14 dias dos compósitos formados por cimento, biomassa e escória de alto-forno. Os resultados dos ensaios de inibição demonstraram que o percentual de $50 \%$ de escória de foi o melhor para a redução da alcalinidade da matriz cimentícia e, consequentemente, aumento da compatibilidade entre a biomassa de bambu e o cimento Portland. Para a resistência à compressão, os teores de 25 a $70 \%$ apresentaram melhora desta propriedade, demonstrando, assim, que a adição de escória de alto-forno aumentou a compatibilidade entre o cimento e a biomassa de bambu. Concluindo-se que esta pode ser utilizada para a confecção de compósitos cimento-biomassa vegetal.

Palavras-chave: Compósitos. Compatibilidade. Escória de alto-forno.

\section{ABSTRACT}

The objective of this research was to evaluate the effect of the addition of different blast furnace slag contents to composite with mosso bamboo (Phyllostachys pubescens), in order to verify the compatibility between biomass, Portland cement and high- oven. The degree of compatibility was determined by the study of the heat of hydration in the first 24 hours and the resistance to axial compression after 14 days of the composites formed by cement, biomass and blast furnace slag. The results of the inhibition tests showed that the 50\% percentage of slag was the best for reducing the alkalinity of the cement matrix and, consequently, increasing the compatibility between bamboo biomass and Portland cement. For the compressive strength, the contents of 25 to $70 \%$ showed improvement of this property, demonstrating, therefore, that the addition of blast furnace slag increased the compatibility between cement and bamboo biomass. It is concluded that this can be used for the preparation of cement-biomass composites.

Keywords: Composites. Compatibility. Blast furnace slag.

\section{INTRODUÇÃO}

Materiais compósitos são aqueles que possuem ao menos dois materiais de naturezas diferentes. A junção destes resulta em um material com propriedades combinadas e superiores, quando comparadas com os materiais originais, sendo composto basicamente por uma matriz e reforço.

Os painéis de cimento madeira são objeto de estudo de vários pesquisadores devido as suas características como: durabilidade, estabilidade dimensional, resistência à absorção de água, rigidez, resistência ao 
fogo, bom isolante acústico e térmico, resistência à degradação biológica, baixo custo de produção, além de auxiliar na reutilização de resíduos de madeira [1] [2] [3].

Segundo Cheumani et al. [4] os compósitos cimento-madeira também podem ser preparados a partir de fibras agrícolas não madeireiras, como exemplo, o sisal, o bambu, o linho, e outros resíduos lignocelulósicos, como por exemplo, bagaço, palha de trigo, jornal, entre outros. Estes resíduos não madeireiros e lignocelulósicos são denominados biomassa.

Este tipo de compósito que apresenta biomassa em sua composição é denominado compósito de biomassa vegetal-cimento, uma série de propriedades se torna inerente com a utilização da biomassa, como comportamento mecânico, baixa massa específica, baixa condutividade térmica, resistência ao fogo, isolamento térmico e impermeabilidade [5].

O bambu vem sendo utilizado devido às vantagens como: o crescimento rápido, fácil acessibilidade, propriedades mecânicas, entre outras. Do ponto de vista físico-mecânico, quando incorporado em painéis apresenta vantagens como aumento das propriedades de resistência, elasticidade e estabilidade dimensional [6].

Porém estes apresentam uma dificuldade para a sua produção: os componentes orgânicos das fibras afetam o processo de hidratação e cura do cimento. Esse efeito inibitório que algumas espécies de madeira exercem no cimento deve-se ao teor e o tipo de extrativos que estas possuem [7] [8].

Os cristais de hidróxido de cálcio $\left(\mathrm{Ca}(\mathrm{OH})_{2}\right)$, representam 20 a $25 \%$ do volume de sólidos na pasta de cimento hidratada. Apesar de não contribuir significativamente para à resistência da pasta, este é responsável pela elevada alcalinidade da matriz cimentícia, $\mathrm{pH}$ de 12,5 [9].

Os componentes da madeira podem ser degradados pelo hidróxido de cálcio formado durante a hidratação do cimento. Este ambiente alcalino é capaz de remover parte dos extrativos e dissolver componentes da madeira como as hemiceluloses, causando assim, alterações dimensionais no compósito [10] [11].

Os carboidratos da madeira reagem com o cálcio, alumínio e cátions de ferro, retardando, assim, a reação de hidratação do cimento, reduzindo sua cristalinidade e resistência [12]. A água presente nos poros da matriz cimentícia apresenta um $\mathrm{pH}$ elevado, sendo capaz de quebrar as ligações químicas das fibras de madeira, possibilitando assim, a migração das partículas de madeira para a superfície da pasta [13] [14].

Outro aspecto a ser considerado é o processo de produção do cimento, no qual são lançados 5\% de dióxido de carbono $\left(\mathrm{CO}_{2}\right)$ do planeta, liberando também óxido de enxofre, óxido de nitrogênio, monóxido de carbono e compostos de chumbo, substâncias poluentes da atmosfera. Para a produção de uma tonelada de cimento, é liberada, em média, uma tonelada de $\mathrm{CO}_{2}$ na atmosfera [15].

Tem-se utilizado materiais alternativos para a substituição do cimento, como exemplo, a escória granulada de alto forno já vem sendo usada como adição ao cimento Portland; quando moída, e tem-se mostrado um material que melhora o desempenho do cimento principalmente em idades tardias [16].

Escórias ácidas normalmente são aquelas produzidas em fornos a carvão vegetal, classificadas dessa forma por conterem em sua composição química sílica como componente predominante e, relação entre seus elementos principais $\mathrm{CaO} / \mathrm{SiO}_{2}$ ser menor que um [17] [18] [19].

As escórias ácidas, diferentemente das escórias básicas, normalmente não apresentam características químicas favoráveis à adição ao cimento Portland de acordo com os índices ou módulos prescritos na norma Brasileira. Dessa forma, elas são então descartadas pela indústria cimenteira podendo-se tornar um passivo ambiental se não for destinada a outros usos [18].

Uma das razões para a adição de escória ao cimento é o fato de esta reduzir o calor liberado durante o processo de hidratação [20], além do apelo sustentável uma vez que sua utilização reduz a energia equivalente agregada no produto final.

A escória granulada de alto-forno apresenta uma taxa de hidratação mais lenta, quando misturada a água [16] [20]. Mas quando na presença do cimento, a escória depende da desagregação e dissolução da sua estrutura vítrea por íons hidroxila, liberados durante a hidratação do cimento Portland, e também do teor de álcalis no cimento. Quando a escória é misturada à água, a sua hidratação consume hidróxido de cálcio presente no cimento Portland e usa este para a formação de gel C-S-H (silicato de cálcio hidratado) adicional [16].

Segundo John e Agopyan [21] o emprego de materiais cimentícios alternativos, como o caso da escória de alto-forno, podem colaborar para um aumento da durabilidade de compósitos com fibras vegetais.

Lange et al. [22], cita os seguintes benefícios causados pela substituição parcial do cimento: redução 
da alcalinidade da matriz cimentícia, alteração da porosidade do material, a matriz cimentícia apresentou menos sensibilidade aos efeitos inibitórios da madeira, aumento da resistência diante do uso de madeiras menos compatíveis com o cimento Portland, aumento da durabilidade, devido a redução do pH da matriz, melhores características diante da umidade, menor retração do material, etc.

Assim esta pesquisa tem por objetivo avaliar o efeito da adição de diferentes teores escória de altoforno à carvão vegetal em compósitos com bambu mossô (Phyllostachys pubescens).

\section{MATERIAIS E MÉTODOS}

Foi utilizado biomassa vegetal de bambu mossô (Phyllostachys pubescens Mazel ex J.Houz) proveniente da cidade de São José dos Pinhais (Paraná, Brasil). Este material é recebido na empresa, e transformado em partículas com o auxílio de moinhos de martelos, após ocorre a classificação de sua granulometria. O tamanho máximo das partículas é de 1,18 mm de acordo com NBR NM 248 [23]. A Figura 1 apresenta as partículas da biomassa.

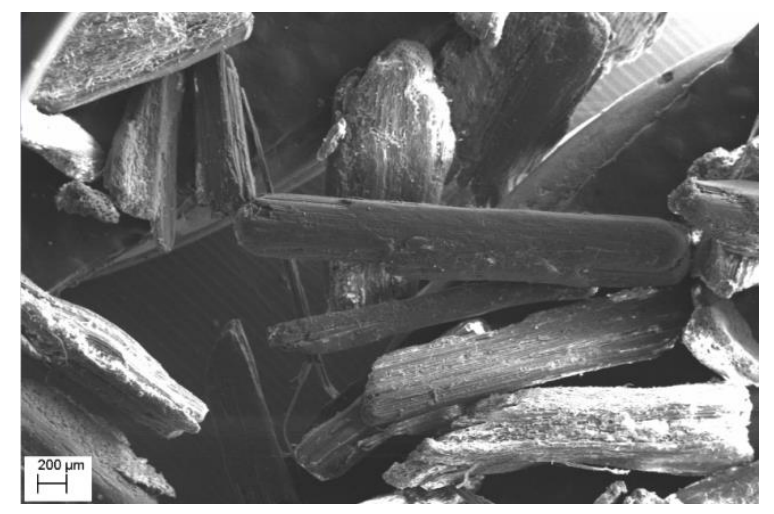

Figura 1: Microscopia eletrônica de varredura (MEV) das fibras da biomassa de bambu mossô (50x)

O aglomerante utilizado foi o Cimento Portland de alta resistência inicial CPV-ARI [24], este foi escolhido devido ao seu tempo de início de pega ser mais rápido que os demais cimentos Portland e alcançar altos níveis de resistência nos primeiros dias após a aplicação. Foi utilizado escória de alto-forno à carvão vegetal proveniente do processo de produção do ferro-gusa.

A água utilizada é da rede de abastecimento da Companhia de Saneamento do Paraná (SANEPAR), sendo o trabalho desenvolvido na cidade de Curitiba - PR.

\subsection{Caracterização da escória de alto-forno}

A escória de alto-forno granulada à carvão vegetal foi seca em estufa, por aproximadamente 24 horas, até atingir a constância do peso, em temperatura controlada de $105^{\circ} \mathrm{C}$, e triturada em moinho de panela. Após isso, passou-se para a caracterização desta, foram seguidos os ensaios citados abaixo:

-Determinação da superfície específica utilizou-se a NBR NM 76: Cimento Portland - Determinação da finura pelo método de permeabilidade ao ar (Método de Blaine) [25];

-A análise química semi quantitativa foi realizada por meio de espectroscopia por fluorescência de Raios X (FRX). Assim, foi possível determinar o Índice de basicidade ou hidraulicidade da escória, onde $\mathrm{CaO} / \mathrm{SiO}_{2} \geq 1$, básica e $\mathrm{CaO} / \mathrm{SiO}_{2}<1$, ácida, e;

-A difração de Raios X (DRX) foi utilizada para fazer a caracterização mineralógica da escória. Utilizou-se o difratômetro de Raios X Shimadzu XRD-7000.

Visando analisar a influência de diferentes teores de escória de alto-forno nos compósitos de cimento com bambu mossô, foram realizados ensaios de inibição e resistência à compressão dos compósitos.

\subsection{Ensaio de inibição}

Para verificar como a composição da escória de alto-forno e a biomassa do bambu afetavam o processo de hidratação do cimento, foi realizado o ensaio de inibição para os teores de escória de alto-forno de: 5, 10, 15, 
25, 30, 40, 50, 60 e 70\%. Realizou-se o ensaio em uma adaptação de calorímetro, como aparelho aquisitor de dados Master Logger A 202 da marca WARME, termopar tipo K (níquel - cromado) ANSI 2 x 24 (0,51mm) Awg em silicone com 8 canais.

Foram moldados três corpos de prova para cada traço, contendo $300 \mathrm{~g}$ de cimento Portland CPV-ARI, 22,5 g de biomassa de bambu tratada com água fria, $150 \mathrm{~g}$ de água, e com os teores de escória de alto-forno de: $0,5,10,15,25,30,40,50,60$ e $70 \%$ em relação a massa do cimento, buscando analisar os efeitos da adição de escória de alto-forno e da biomassa sobre o compósito. Também foram moldados três corpos de prova da pasta referência (300 g de cimento e $120 \mathrm{~g}$ de água). Após a mistura, estes foram colocados em um recipiente térmico $(5 \times 10 \mathrm{~cm})$, onde o cabo de compensação tipo K2x24 com isolamento de silicone foi introduzido. O cabo de compensação encontrava-se conectado a um receptor de sinais, no qual os dados eram lidos e convertidos em valores de temperatura por um programa de computador. As leituras foram coletadas a cada segundo por um período de 24 horas. Este método foi baseado na metodologia desenvolvida por Hofstrand et al [26].

A partir das curvas de hidratação, foi possível determinar a temperatura máxima atingida por estes, o tempo em que esta temperatura foi atingida e a taxa máxima de variação da temperatura pelo tempo, permitindo, assim, determinar o índice de inibição para os compósitos.

Para o cálculo do índice de inibição, foi utilizada a equação elaborada por Hofstrand et al. [26] conforme indicado na Equação (1):

$$
\mathrm{I}=\left[\left(\frac{\mathrm{t} 2-\mathrm{t}^{\prime} 2}{\mathrm{t}^{\prime} 2}\right)\left(\frac{\mathrm{T}^{\prime} 2-\mathrm{T} 2}{\mathrm{~T}^{\prime} 2}\right)\left(\frac{\mathrm{S}^{\prime}-\mathrm{S}}{\mathrm{S}^{\prime}}\right)\right] * 100
$$

Em que:

t2: tempo para atingir a máxima temperatura (cimento-madeira-água);

T2: temperatura máxima de hidratação (cimento-madeira-água);

S: taxa máxima de variação de temperatura no decorrer do tempo (cimento-madeira-água);

t’2: tempo para atingir a máxima temperatura (pasta);

T’2: temperatura máxima de hidratação (pasta), e;

S': taxa máxima de variação de temperatura no decorrer do tempo (pasta).

Segundo a Equação (1) a madeira pode ser classificada em quatro categorias, conforme Tabela 1 [27].

Tabela 1: Classificação da inibição da hidratação do cimento segundo o índice de inibição.

\begin{tabular}{l|l}
\hline ÍNDICE DE INIBIÇÃO & CLASSIFICAÇÃO \\
\hline $\mathrm{I}<10$ & Baixa inibição \\
\hline $\mathrm{I}=10$ a 50 & Moderada inibição \\
\hline $\mathrm{I}=50$ a 100 & Alta inibição \\
\hline $\mathrm{I}>100$ & Extrema inibição \\
\hline
\end{tabular}

\subsection{Ensaio mecânico}

A moldagem dos corpos de prova cilíndricos foi feita em moldes de PVC com dimensão de 1,6 por 3,2 cm, obedecendo a relação estabelecida pela NBR 7222 [28], que define a relação entre o diâmetro do molde e a sua altura seja de 1:2. Os corpos-de-prova foram moldados realizando o preenchimento em três camadas e 30 quedas manuais, de $2 \mathrm{~cm}$ de altura, visando a expulsão do ar das amostras, bem como o correto adensamento das camadas, com base na NBR 7215 [29]. O traço referência era composto por uma pasta de cimento:água.

O ensaio de resistência à compressão realizou-se em uma máquina universal de ensaios DL10.000 da marca EMIC. Para cada amostra foram ensaiados 6 corpos-de-prova aos quatorze dias de idade. Os traços ensaiados são apresentados na Tabela 2. 
Tabela 2: Traços dos compósitos com escória de alto-forno à carvão vegetal

\begin{tabular}{l|l|l|l}
\hline TRAÇO & $\begin{array}{l}\text { RELAÇÃO CIMENTO:BIOMASSA } \\
\text { DE BAMBU (Traços em massa) }\end{array}$ & $\begin{array}{l}\text { RELAÇÃO } \\
\text { ÁGUA:CIMENTO }\end{array}$ & ESCÓRIA (\%) \\
\hline T1 & $1: 13$ & 0,5 & 5 \\
\hline T2 & $1: 13$ & 0,5 & 10 \\
\hline T3 & $1: 13$ & 0,5 & 15 \\
\hline T4 & $1: 13$ & 0,5 & 25 \\
\hline T5 & $1: 13$ & 0,5 & 30 \\
\hline T6 & $1: 13$ & 0,5 & 40 \\
\hline T7 & $1: 13$ & 0,5 & 50 \\
\hline T8 & $1: 13$ & 0,5 & 60 \\
\hline T9 & $1: 13$ & 0,5 & 70 \\
\hline $\begin{array}{l}\text { Pasta referên- } \\
\text { cia }\end{array}$ & $1: 0$ & 0,5 & - \\
\hline
\end{tabular}

Os resultados foram analisados estatisticamente, por meio de comparação múltipla entre médias pelo teste de Tukey com nível de confiança de $95 \%$.

\section{RESULTADOS E DISCUSSÕES}

\subsection{Caracterização da escória de alto-forno}

\subsubsection{Distribuição granulométrica e Finura Blaine}

A superfície específica da escória de alto-forno foi determinada pelo método de Blaine, apresentando valor de $3728,83 \mathrm{~cm}^{2} / \mathrm{g}$. Dessa forma conclui-se que a escória atende as especificações da norma britânica BS 6699: Specification for Ground granulated blastfurnace slag for use with Portland cement [30], que estabelece que a escória deve possuir finura/superfície específica superior a $2750 \mathrm{~cm}^{2} / \mathrm{g}$, já a ASTM C 989: Standard Specification for Slag Cement for Use in Concrete and Mortars [31] estabelece que as escórias devem possuir superfície específica de $4950 \mathrm{~cm}^{2} / \mathrm{g}$, assim, a escória adotada não atende a norma americana.

Observa-se pelos resultados encontrados que a superfície específica da escória $\left(3728,83 \mathrm{~cm}^{2} / \mathrm{g}\right)$ se aproxima da superfície específica do cimento CPV - ARI, que é de $4320 \mathrm{~g} / \mathrm{cm}$. Esse resultado demonstra que há compatibilidade entre as granulometrias, o que permite sua utilização como adição ao cimento Portland. Ainda, a escória de alto-forno e outros materiais, com os mesmos constituintes, podem ser adicionados ao cimento, pois também reagem com o hidróxido de cálcio dando origem a géis de silicatos $(\mathrm{C}-\mathrm{S}-\mathrm{H})$, os quais são os principais responsáveis pela resistência mecânica da matriz conforme demostrado por Pal et al [16].

\subsubsection{Espectroscopia por fluorescência de Raios $\mathrm{X}$}

A análise química semi-quantitativa por espectroscopia por fluorescência de raios X é apresentada na Tabela 3 .

Tabela 3: Composição química da escória de alto-forno à carvão vegetal

\begin{tabular}{l|l}
\hline COMPONENTE & TEOR (\%) \\
\hline $\mathrm{SiO}_{2}$ & 39,7 \\
\hline $\mathrm{CaO}$ & 32,8 \\
\hline $\mathrm{Al}_{2} \mathrm{O}_{3}$ & 12,9 \\
\hline $\mathrm{MgO}$ & 7,2 \\
\hline
\end{tabular}




\begin{tabular}{|c|c|}
\hline $\mathrm{Fe}_{2} \mathrm{O}_{3}$ & 2,6 \\
\hline $\mathrm{TiO}_{2}$ & 0,7 \\
\hline $\mathrm{K}_{2} \mathrm{O}$ & 0,7 \\
\hline $\mathrm{MnO}$ & 0,6 \\
\hline $\mathrm{Na}_{2} \mathrm{O}$ & 0,1 \\
\hline $\mathrm{SrO}$ & 0,1 \\
\hline $\mathrm{SO}_{3}$ & 0,1 \\
\hline $\mathrm{ZrO}_{2}$ & 0,1 \\
\hline $\mathrm{Cr}_{2} \mathrm{O}_{3}$ & $<0,1$ \\
\hline $\mathrm{P}_{2} \mathrm{O}_{5}$ & $<0,1$ \\
\hline Perda ao Fogo & 2,35 \\
\hline $\mathrm{CaO} / \mathrm{SiO}_{2}$ & 0,83 \\
\hline $\begin{array}{l}\mathrm{CaO}+\mathrm{MgO}+\mathrm{Al}_{2} \mathrm{O}_{3} / \mathrm{SiO}_{2}>1,0 \\
\mathrm{NBR} 5735[32]\end{array}$ & 1,33 \\
\hline $\begin{array}{l}\left(\mathrm{CaO}+\mathrm{SiO}_{2}+\mathrm{MgO}\right) \geq 2 / 3 \text { total } \\
\mathrm{EN} 197-1[33] \text { e BS } 6699[30]\end{array}$ & 79,7 \\
\hline
\end{tabular}

Observa-se que a escória é composta por pelo menos $2 / 3$ da soma dos óxidos de cálcio $(\mathrm{CaO})$, silício $\left(\mathrm{SiO}_{2}\right)$ e magnésio $(\mathrm{MgO})$ da massa total dos óxidos que a compõem, o que segundo a norma europeia EN 197-1 [33] e a norma britânica BS 6699 [30] é um requisito para a utilização de escória em cimentos de alto forno. No que se refere a NBR 5735 [32], está também cumpre o determinado referente à relação entre seus principais componentes.

Pela relação $\mathrm{CaO} / \mathrm{SiO}_{2}$, a qual classifica escórias como ácidas $(\mathrm{C} / \mathrm{S}<1)$ e básicas $(\mathrm{C} / \mathrm{S}>1)$, esta escória apresenta valor de 0,83 , sendo classificada como ácida.

\subsubsection{Difração de Raios X}

A Figura 2 apresenta o difratograma da escória de alto-forno. 


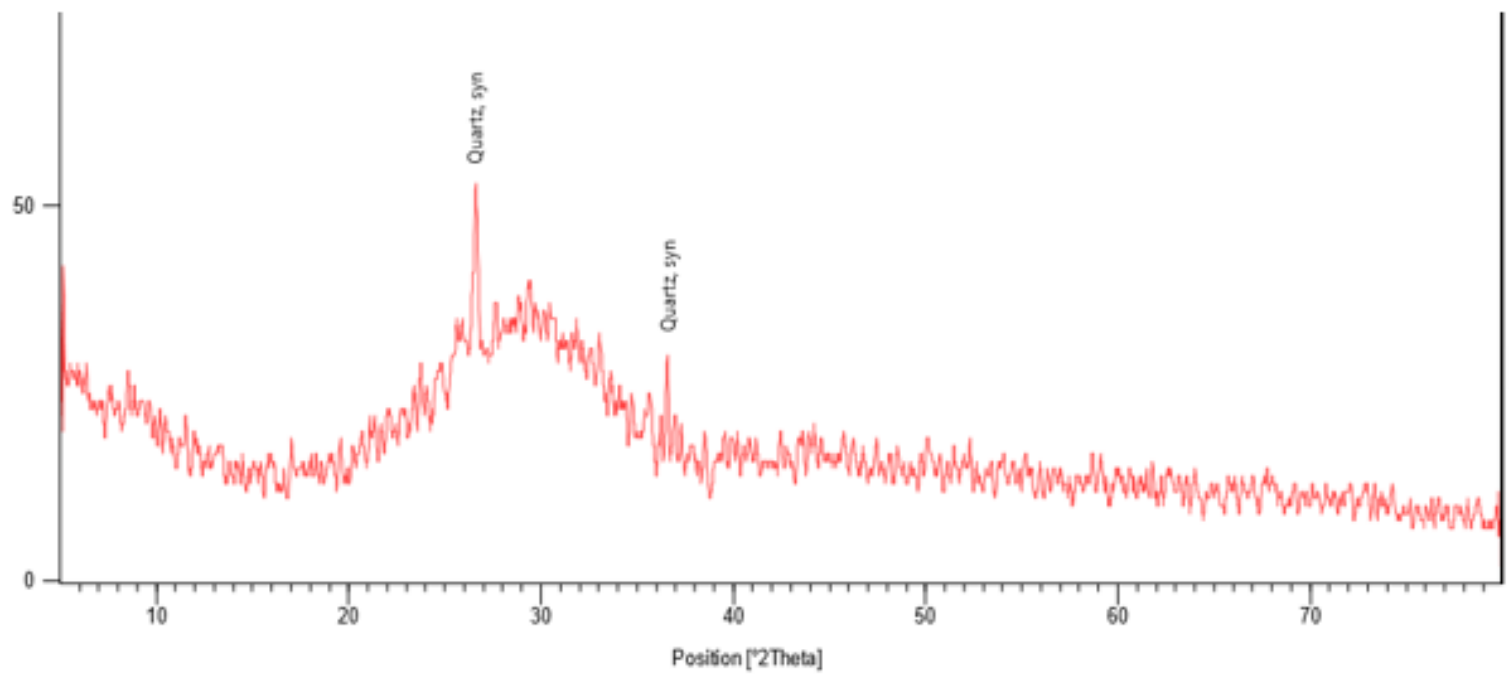

Figura 2: Difração de Raios X para a escória de alto-forno à carvão vegetal

Através da Figura 2, é possível observar que a escória apresenta dois picos de quartzo $\left(\mathrm{SiO}_{2}\right)$, e através da análise do difratograma desta, verifica se que esta é um material amorfo, devido ao fato de não apresentar picos característicos e dessa forma a ausência de um arranjo ordenado e periódico de átomos.

Além disso, o dióxido de silício ( sílica $-\mathrm{SiO}_{2}$ ) tende a reagir com o hidróxido de cálcio presente no cimento, e formar uma quantidade extra de silicato de cálcio hidratado, responsável pelo aumento da resistência à compressão na matriz cimentícia. Justificando a adição de escória de alto-forno ao compósito.

\subsection{Ensaio de inibição}

A Figura 3 apresenta as curvas de hidratação para os compósitos com adição de escória de alto-forno.

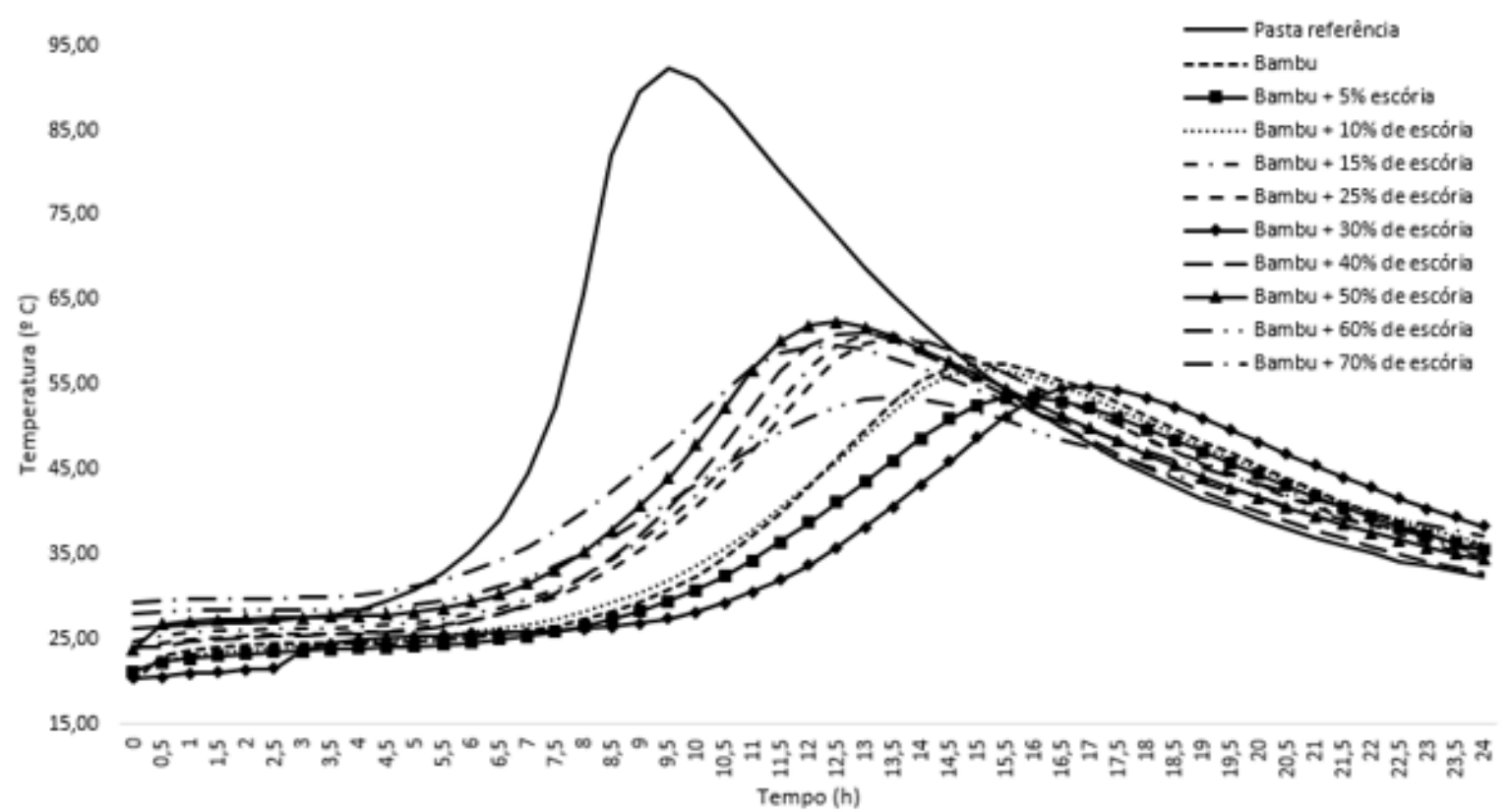

Figura 3: Curvas de hidratação da pasta, e do compósito a base de biomassa de bambu mossô e adição de escória de altoforno à carvão vegetal 
Com base na Figura 3, observa-se que quando comparados com a pasta referência, nenhum compósito conseguiu atingir a temperatura máxima de hidratação do cimento CPV-ARI, ficando todos abaixo desta. Isto ocorre devido ao fato da biomassa de bambu, mesmo quando tratada com água fria e com a adição de escória de alto-forno, interferiu nas reações de hidratação do cimento Portland, reduzindo seu calor de hidratação. Pois parte dos extrativos, ainda permanece na biomassa reduzindo a velocidade de hidratação do cimento. A redução dessa velocidade implica na redução da taxa de liberação de calor, lembrando que esta é uma reação exotérmica. Também observou-se que os compósitos demoram mais tempo para atingir a máxima temperatura de hidratação do cimento, demonstrando assim, a influência do bambu mossô na hidratação deste.

A Tabela 4 apresenta os valores de temperatura máxima, tempo mínimo, índice de inibição e classificação quanto ao índice de inibição para os compósitos de bambu mossô com a adição de escória de alto-forno à carvão vegetal.

Tabela 4: Valores médios de temperatura, tempo e índice de inibição para o compósito com farinha de bambu mossô tratada com água fria e escória de alto-forno à carvão vegetal

\begin{tabular}{|c|c|c|c|c|}
\hline & $\begin{array}{l}\text { TEMPERATURA } \\
\text { MÁXIMA }\left({ }^{\circ} \mathrm{C}\right)\end{array}$ & TEMPO MÍNIMO (h) & I (\%) & $\begin{array}{l}\text { CLASSIFICAÇÃO } \\
\text { QUANTO AO ÍNDICE } \\
\text { DE INIBIÇÃO }\end{array}$ \\
\hline Pasta Padrão & $92,33^{\mathrm{A}}$ & $9,5^{\mathrm{H}}$ & - & - \\
\hline $\begin{array}{l}\text { Bambu mossô } \\
\text { (Água fria) }\end{array}$ & $57,33^{\mathrm{B}}$ & $15,5^{\mathrm{C}}$ & $16,42^{\mathrm{C}}$ & Moderada inibição \\
\hline $\begin{array}{l}\text { Bambu mossô }+5 \% \\
\text { de escória }\end{array}$ & $53,53^{\mathrm{B}}$ & $16^{\mathrm{B}}$ & $21,91^{\mathrm{B}}$ & Moderada inibição \\
\hline $\begin{array}{l}\text { Bambu mossô + } \\
10 \% \text { de escória }\end{array}$ & $56,77^{\mathrm{B}}$ & $15^{\mathrm{D}}$ & $15,32^{\mathrm{D}}$ & Moderada inibição \\
\hline $\begin{array}{l}\text { Bambu mossô + } \\
15 \% \text { de escória }\end{array}$ & $60,93^{\mathrm{B}}$ & $13^{\mathrm{E}}$ & $6,76^{\mathrm{G}}$ & Baixa inibição \\
\hline $\begin{array}{l}\text { Bambu mossô + } \\
25 \% \text { de escória }\end{array}$ & $60,40^{\mathrm{B}}$ & $13,5^{\mathrm{E}}$ & $7,99^{\mathrm{F}}$ & Baixa inibição \\
\hline $\begin{array}{l}\text { Bambu mossô + } \\
30 \% \text { de escória }\end{array}$ & $54,73^{\mathrm{B}}$ & $17^{\mathrm{A}}$ & $27,44^{\mathrm{A}}$ & Moderada inibição \\
\hline $\begin{array}{l}\text { Bambu mossô + } \\
40 \% \text { de escória }\end{array}$ & $61,07^{\mathrm{B}}$ & $13^{\mathrm{F}}$ & $6,13^{\mathrm{H}}$ & Baixa inibição \\
\hline $\begin{array}{l}\text { Bambu mossô + } \\
50 \% \text { de escória }\end{array}$ & $62,40^{\mathrm{B}}$ & $12,5^{\mathrm{G}}$ & $4,91^{\mathrm{J}}$ & Baixa inibição \\
\hline $\begin{array}{l}\text { Bambu mossô + } \\
60 \% \text { de escória }\end{array}$ & $53,45^{\mathrm{B}}$ & $13,5^{\mathrm{E}}$ & $11,53^{\mathrm{E}}$ & Moderada inibição \\
\hline $\begin{array}{l}\text { Bambu mossô + } \\
70 \% \text { de escória }\end{array}$ & $59,55^{\text {B }}$ & $12,5^{\mathrm{G}}$ & $6,09^{\mathrm{I}}$ & Baixa inibição \\
\hline
\end{tabular}

*Valores médios de três repetições

**Médias seguidas pela mesma letra dentro da mesma coluna não se diferenciam estatisticamente, a 5\% de probabilidade, pelo teste de Tukey.

Com base nos índices de inibição, verifica-se que a escória de alto-forno interfere de forma positiva na hidratação do cimento, como exemplo, a adição de $50 \%$ de escória ao compósito que acabou por influenciar positivamente o índice de inibição, alterando este de moderada inibição, para o compósito feito somente com bambu, para baixa inibição, quando é adicionado 50\% de escória ao compósito.

Ainda levando em consideração estes dois compósitos, no que se refere a temperatura máxima de hidratação, pôde-se verificar que há um aumento nesta de 8,00\%. No que se refere ao tempo mínimo, verifica-se que ocorre uma redução de $19 \%$ para atingir a máxima temperatura de hidratação do cimento, sendo este comportamento também observado para os compósitos com $10 \%$ e $25 \%$ de escória. 
Porém, há composições em que a adição de escória acabou por influenciar negativamente no índice de inibição e na temperatura de hidratação do cimento, como exemplo, os compósitos com adição de 5\% e 30\% de escória, também pôde-se atentar para o fato de que nestes dois compósitos há um aumento de 3,00\% e 9,00\%, respectivamente, no tempo mínimo para atingir a máxima temperatura de hidratação do cimento.

Constatou-se como resultado que a escória de alto-forno auxiliou no aumento da compatibilidade dos compósitos. Isto ocorre devido ao fato de que a escória apresenta um alto teor de silíca em sua composição, esta quando em contato com a matriz cimentícia reage com o hidróxido de cálcio, diminuindo a alcalinidade dos compósitos.

Através do teste Tukey, pode-se afirmar que não há diferenças significativas para as médias da temperatura de hidratação dos compósitos e que há diferenças significativas para o tempo mínimo e o índice de inibição dos compósitos.

\subsection{Ensaio mecânico}

A Tabela 5 apresenta os valores obtidos para a resistência à compressão dos corpos de prova moldados com a biomassa de bambu mossô e com a adição de escória de alto-forno, para estes a relação água:cimento foi de 0,5 .

Tabela 5: Resistência à compressão dos compósitos feitos com escória de alto-forno com relação água:cimento =0,5

\begin{tabular}{|c|c|c|c|}
\hline TRAÇO & ESCÓRIA (\%) & $\begin{array}{l}\text { RESISTÊNCIA À COMPRESSÃO } \\
\text { (MPA) }\end{array}$ & $\begin{array}{l}\text { COEFICIENTE DE VARI- } \\
\text { AÇÃO (\%) }\end{array}$ \\
\hline $\mathrm{T} 1$ & 5 & $9,83^{\mathrm{C}}$ & 17,60 \\
\hline $\mathrm{T} 2$ & 10 & $13,64^{\mathrm{BC}}$ & 12,58 \\
\hline $\mathrm{T} 3$ & 15 & $13,67^{\mathrm{BC}}$ & 13,14 \\
\hline $\mathrm{T} 4$ & 25 & $15,62^{\mathrm{B}}$ & 14,37 \\
\hline T5 & 30 & $14,28^{\mathrm{B}}$ & 10,77 \\
\hline T6 & 40 & $17,61^{\mathrm{B}}$ & 5,20 \\
\hline $\mathrm{T} 7$ & 50 & $15,81^{\mathrm{B}}$ & 7,70 \\
\hline $\mathrm{T} 8$ & 60 & $16,21^{B}$ & 10.05 \\
\hline T9 & 70 & $16,97^{\mathrm{B}}$ & 18,08 \\
\hline Pasta referência & - & $23,28^{\mathrm{A}}$ & 18,31 \\
\hline
\end{tabular}

Através da Tabela 5 observa-se que mesmo com o aumento do teor de escória de alto-forno na composição não afetou a resistência à compressão dos compósitos, sendo que estes foram elevando sua resistência conforme incorporou-se escória a mistura. Assim, pôde-se afirmar que não há diferença significativa para as médias da resistência à compressão dos compósitos dos traços T2 a T9, levando-se em consideração o teste Tukey.

Este aumento da resistência à compressão dos compósitos com substituição do cimento Portland pela escória de alto-forno, ocorre devido ao fato de que a escória apresenta um alto teor de sílica em sua composição, esta quando em contato com a matriz cimentícia reage com o hidróxido de cálcio presente na pasta, formando uma quantidade extra de silicato de cálcio hidratado, sendo este o principal responsável pelo aumento da resistência à compressão da matriz cimentícia.

\section{CONCLUSÕES}

Analisando-se o efeito da adição de escória de alto-forno sobre o índice de inibição dos compósitos e sobre a resistência à compressão destes, observa-se que mesmo variando o teor de escória de alto-forno de 5\% a 70\%, estes não afetam de forma negativa a compatibilidade do sistema. Para o índice de inibição, verifica-se que o 
teor de 50\% de escória de alto-forno, foi o que mais aumentou a compatibilidade entre a biomassa do bambu mossô e o cimento Portland. Isso ocorre devido a redução da alcalinidade da matriz cimentícia, possibilitando, assim, uma melhor interação da biomassa com a matriz cimentícia e, consequentemente, menor interferência nas reações de hidratação do cimento.

Para a resistência à compressão, verifica-se que os teores de 25 a $70 \%$ de escória de alto-forno, auxiliaram no aumento desta propriedade, isto se deve ao fato de que ocorre um aumento da quantidade de silicato de cálcio hidratado, devido a reação entre sílica da escória com o hidróxido de cálcio do cimento, sendo o silicato de cálcio o principal responsável pela resistência à compressão da matriz cimentícia.

Verifica-se a viabilidade de substituição do cimento portland pela escória de alto-forno, pois o cimento é um aglomerante que causa grandes emissões de $\mathrm{CO} 2$ na atmosfera, além de apresentar valor comercial superior a escória de alto-forno, sendo esta um resíduo na produção de ferro gusa, apresentando grande viabilidade econômica para sua utilização.

\section{AGRADECIMENTOS}

Os autores agradecem a Fundação Araucária e a CAPES pelo seu apoio.

\section{BIBLIOGRAFIA}

[1] DEL MENEZZI, C. H. S., CASTRO, V. G., SOUZA, M. R. "Production and properties of a medium density wood-cement boards produced with oriented strands and silica fume", Maderas Ciencia y tecnologia. v. 9, n. 2, pp. 105-115, 2007.

[2] PEHANICH, J. L., BLANKENHORN, P. R., SILSBEE, M. R. "Wood fiber surface treatment level effects on selected mechanical properties of wood fiber-cement composites", Cement and Concrete Research, v. 34, n. 1, pp. 59-65, 2004.

[3] FAN, M., NDIKONTAR, M. K., ZHOU, X., et al., "Cement-bonded composites made from tropical woods: Compatibility of wood and cement”, Construction and Building Materials, v. 36, pp. 135-140, 2012.

[4] CHEUMANI, Y. A. M., NDIKONTAR, M., JÉSO, B., et al., "Probing of wood-cement interactions during hydration of wood-cement composites by proton low-field NMR relaxometry", Journal of Materials Science, v. 46, n. 5, pp. 1167-1175, 2011.

[5] PIMENTEL, L., BERALDO, A. L., JÚNIOR, H. S. "Durabilidade de compósito biomassa vegetalcimento modificado por polímero", Eng. Agrícola [Online], v. 26, n. 2, pp. 344-353, 2006.

[6] HIZIROGLU, S., JARUSOMBUTI, S., FUEANGVIVAT, V., et al., "Properties of bamboo-rice straweucalyptus composite panels", Forest Products Journal, v.55, pp.221-225, 2005.

[7] JORGE, F. C. PEREIRA, C. FERREIRA, J. M. F. "Wood-cement composites: a review", European Journal of Wood and Wood Products, v. 62, n. 5, pp. 370-377, 2004.

[8] MACÊDO, A. N., SOUZA, A. A. C., NETO, B. B. P. "Chapas de cimento-madeira com resíduos da indústria madeireira da Região Amazônica”, Ambiente Construído, v. 12, n. 2, pp. 131-150, 2012.

[9] SENFF, L., FOLGUERAS, M. V., HOTZA, D. "Hidratação do cimento CP V ARI-RS: Influência da água nas reações de hidratação”, In: Congresso brasileiro de cerâmica. São Paulo, 2005.

[10] MILLER, D. P., MOSLEMI, A. A. "Wood-cement composites: effect of model compounds on hydration characteristics and tensile strength", Wood and Fiber Science, v. 23, n. 4, pp. 472-482, 1991.

[11] FAN, M. Z., BONFIELD, P. W., DINWOODIE, J. M., et al., "Dimensional instability of cementbonded particleboard: Mechanisms of deformation of CBPB", Cement and Concrete Research, v. 29, n. 6, pp. 923-932, 1999.

[12] HACHMI, M. H., CAMPBELL, A. G. "Wood-cement chemical relationships, Fiber and Particleboards Bonded with Inorganic Binders”, Forest Products Research Society. Madison, pp. 43-47, 1989.

[13] YOUNG. J. F. "Effect of organic compounds on the interconversions of calcium aluminate hydrates: hydration of tricalcium aluminate", Journal of the american ceramic society, v. 53, n. 2, pp. 65-69, 1970.

[14] SILVA, A. C., JUNIOR, H. S., JOHN, V. M. "Envelhecimento de compósitos à base de escória de altoforno reforçados com polpa celulósica residual de eucalipto", Ambiente Construído, v. 9, n. 1, pp. 25-44, 
2009.

[15] AITCIN, P. C., MINDESS, S. Sustainability of Concrete. USA e Canada: Spon Press, 2011.

[16] PAL, S. C., MUKHERJEE, A., PATHAK, S. R. "Investigation of hydraulic activity of ground granulated blast furnace slag in concrete", Cement and Concrete Research, v. 33, n. 9, pp. 1481-1486, 2003.

[17] JOHN, M. V. Cimentos de escória ativada com silicatos de sódio. Tese (Doutorado), Programa de PósGraduação em Engenharia de Construção Civil da Escola Politécnica da Universidade de São Paulo. São Paulo, 1995.

[18] MASSUCATO, C. J. Utilização de escória de alto-forno à carvão vegetal como adição no concreto. Dissertação de M.Sc, Programa de Pós-Graduação da Universidade Estadual de Campinas, Faculdade de Engenharia Civil, Arquitetura e Urbanismo, Campinas, São Paulo, Brasil, 2005.

[19] MALARD, A. A. M. Avaliação ambiental do setor de siderurgia não integrada a carvão vegetal do estado de Minas Gerais. Dissertação de M.Sc., Programa de Pós-Graduação em Sustentabilidade Socioeconômica e Ambiental. Universidade Federal de Ouro Preto, Ouro Preto, Minas Gerais, Brasil, 2009.

[20] KOLANI, B., LACARRIÈRE-BUFFO, L., SELLIER, A., et al.,"Hydration of slag-blended cements", Cement \& Concrete Composites, v. 34, n. 9, pp. 1009-1018, 2012.

[21] JOHN, V.M., AGOPYAN, V. "Materiais reforçados com fibras vegetais”, In: Simpósio Internacional de Materiais Reforçados com Fibras para a Construção Civil. Anais. 2, pp. 27-38, EPUSP, São Paulo, 1993.

[22] LANGE, H., SIMATUPANG, M. H., NEUBAUER, A. "Influence of latent hydraulic binders on the properties of wood-cement composites", In: $1^{\circ}$ Inorganic Bonded Fiber Composites Symposium. Forest Products Research Society. Proceedings, v. 1, pp. 48-52, USA, 1989.

[23] ASSOCIAÇÃO BRASILEIRA DE NORMAS TÉCNICAS. NBR NM 248: - Agregados - Determinação da composição granulométrica. Rio de Janeiro. 2003, 6 p.

[24] ASSOCIAÇÃO BRASILEIRA DE NORMAS TÉCNICAS. NBR 5733 (EB - 2): Cimento Portland de Alta Resistência Inicial. Rio de Janeiro, 1991, 5 p.

[25] ASSOCIAÇÃO BRASILEIRA DE NORMAS TÉCNICAS. NBR NM 76 - Cimento Portland - Determinação da finura pelo método de permeabilidade ao ar (Método de Blaine). Rio de Janeiro, 1998, 12 p.

[26] HOFSTRAND, A. D., MOSLEMI, A. A., GARCIA, J. F. "Curing characteristics of wood particle from nine northern Rocky Mountain species mixed with Portland cement”, Forest Products Journal, v. 34, n. 2, pp. 57-61, 1984.

[27] OKINO, E. Y. A., SOUZA, M. R., SANTANA, M. A. E., et al., "Physico-mechanical properties and decay resistance of Cupressus spp. cement-bonded particleboards", Cement and Concrete Composites, v. 27, n. 3, pp. 333-338, 2005.

[28] ASSOCIAÇÃO BRASILEIRA DE NORMAS TÉCNICAS. NBR 7222 - Concreto e argamassa - Determinação da resistência à tração por compressão diametral de corpos de prova cilíndricos. Rio de Janeiro, 2011, 5 p.

[29] ASSOCIAÇÃO BRASILEIRA DE NORMAS TÉCNINCAS. NBR 7215 - Cimento Portland - Determinação da resistência à compressão. Rio de Janeiro, 1996, 8 p.

[30] BRITISH STANDARD INSTITUTION. BS 6699. Specification for Ground granulated blastfurnace slag for use with Portland cement. 1992, 22 p.

[31] AMERICAN SOCIETY FOR TESTING AND MATERIALS. ASTM C989/C989M: Standard Specification for Slag Cement for Use in Concrete and Mortars. Estados Unidos, 1999, 8 p.

[32] ASSOCIAÇÃO BRASILEIRA DE NORMAS TÉCNICAS. NBR 5735 - Cimento Portland de alto-forno. Rio de Janeiro, 1991, 6 p.

[33] EUROPEAN STANDARD - Cement - Part 1: Composition, specifications and conformity criteria for common cements. EN, CEN 197-1: 2000. European Committee for Standardization, Brussels, 2000. 